PROCEEDINGS OF THE

AMERICAN MATHEMATICAL SOCIETY

Volume 132, Number 12, Pages 3467-3471

S 0002-9939(04)07438-6

Article electronically published on July 14, 2004

\title{
COPRIME PACKEDNESS AND SET THEORETIC COMPLETE INTERSECTIONS OF IDEALS IN POLYNOMIAL RINGS
}

\author{
V. ERDOĞDU \\ (Communicated by Bernd Ulrich)
}

\begin{abstract}
A ring $R$ is said to be coprimely packed if whenever $I$ is an ideal of $R$ and $S$ is a set of maximal ideals of $R$ with $I \subseteq \bigcup\{M \in S\}$, then $I \subseteq M$ for some $M \in S$. Let $R$ be a ring and $R\langle X\rangle$ be the localization of $R[X]$ at its set of monic polynomials. We prove that if $R$ is a Noetherian normal domain, then the ring $R\langle X\rangle$ is coprimely packed if and only if $R$ is a Dedekind domain with torsion ideal class group. Moreover, this is also equivalent to the condition that each proper prime ideal of $R[X]$ is a set theoretic complete intersection. A similar result is also proved when $R$ is either a Noetherian arithmetical ring or a Bézout domain of dimension one.
\end{abstract}

\section{INTRODUCTION}

Let $R$ be a commutative ring with identity and $R[X]$ be the polynomial ring over $R$. The author in [3] raised the question of which rings $R$ have the property that the polynomial ring $R[X]$ is coprimely packed and showed that for $R$ Noetherian and Hilbert, $R[X]$ is coprimely packed if and only if $R$ is Artinian. In [5] it is shown that if $R$ is Noetherian and $R[X]$ is coprimely packed, then $R$ is semilocal and is of Krull dimension at most one. In [9] it is proved that for a Noetherian ring $R, R[X]$ is coprimely packed if and only if $R$ is semilocal and $\left(R_{M}\right) / \operatorname{nil}\left(R_{M}\right)$ is seminormal for each maximal ideal $M$ of $R$ such that $R / M$ has characteristic zero. Here we continue our study of coprime packedness of polynomial rings and characterize them in terms of class group and set theoretic complete intersection of prime ideals. Denoting the localization of $R[X]$ at its set of monic polynomials by $R\langle X\rangle$, our objective is to prove the following two results:

(1) Over a Noetherian normal domain $R$ the following statements are equivalent.

(a) $R\langle X\rangle$ is coprimely packed.

(b) $R\langle X\rangle$ is a Dedekind domain with torsion ideal class group.

(c) $R$ is a Dedekind domain with torsion ideal class group.

(d) $R$ is of dimension one, and each proper prime ideal of $R[X]$ is a set theoretic complete intersection.

Received by the editors July 17, 2002 and, in revised form, June 25, 2003.

2000 Mathematics Subject Classification. Primary 13B25, 13B30, 13C15, 13C20; Secondary 13A15, $13 \mathrm{~A} 18$.

Key words and phrases. Coprime packedness, polynomial rings, class group, set theoretic complete intersection.

(C)2004 American Mathematical Society 
(2) If either $R$ is a Noetherian arithmetical ring or is a Bézout domain of dimension one, then over $R$ the following statements are equivalent.

(a) $R\langle X\rangle$ is coprimely packed.

(b) $R$ is coprimely packed.

(c) Each proper prime ideal of $R[X]$ is a set theoretic complete intersection.

These results are in contrast with the following situations. If $R$ is a local ring, then it is coprimely packed, but $R\langle X\rangle$ needs not be coprimely packed. For if $K$ is a field and $Y_{1}, Y_{2}$ are two indeterminates, then $R=K\left[\left[Y_{1}, Y_{2}\right]\right]$ is coprimely packed. However, $R\langle X\rangle$ is not coprimely packed. This is because by [1, $R\langle X\rangle$ is a Noetherian Hilbert ring of dimension two, and by Proposition 1 of [3], a coprimely packed Noetherian Hilbert ring is of dimension at most one. Also, if $R$ is coprimely packed, then so is $R(X)$, where $R(X)$ is the localization of $R[X]$ at the set of polynomials with unit content. However, the coprime packedness of $R(X)$ does not imply the same for $R$, even if $R$ is a Dedekind domain [4].

\section{Coprime packedness When the base Ring is Noetherian}

The equivalence of the statements in the following definition is proved in Lemma 2 of $[5]$.

Definition. A ring $R$ is said to be coprimely packed if it satisfies one of the following equivalent conditions.

(i) If $I$ is an ideal in $R$, and if $S$ is a set of prime ideals in $R$ with $I \subseteq \bigcup\{P \in S\}$, then $I+P \neq R$ for some $P \in S$.

(ii) If $I$ is an ideal in $R$, and if $S$ is a set of maximal ideals in $R$ with $I \subseteq$ $\bigcup\{M \in S\}$, then $I \subseteq M$ for some $M \in S$.

(iii) If $I$ is an ideal in $R$, then $I \not \subset \bigcup\{M \mid M$ is a maximal ideal with $I \not \subset M\}$.

(iv) If $P$ is a prime ideal in $R$, and if $S$ is a set of maximal ideals in $R$, with $P \subseteq \bigcup\{M \in S\}$, then $P \subseteq M$ for some $M \in S$.

(v) For each prime ideal $P$ of $R$, there is an element $b \in P$ with $j-\operatorname{rad} R b=$ $j-\operatorname{rad} P$, where the $j$-radical of an ideal $I$ of $R$ is the intersection of all maximal ideals that contain $I$.

Proposition 1.1. Let $R$ be a Noetherian integral domain. If $R\langle X\rangle$ is coprimely packed, then so is $R$.

Proof. Since $R$ is Noetherian, $R\langle X\rangle$ is a Hilbert ring [1. That is, $R\langle X\rangle$ is a coprimely packed Noetherian Hilbert ring and hence by Proposition 1 of $[3$, it is of dimension at most one. Now by 8 , Corollary 2.2], we have $\operatorname{dim} R=\operatorname{dim} R\langle X\rangle$, and so $R$ is of dimension at most one. If $R$ is zero dimensional, then it is a field, and hence is coprimely packed. So suppose that $R$ is of dimension one, and let $M$ be any non-zero prime ideal of $R$. Then $M R\langle X\rangle$ is a maximal ideal of $R\langle X\rangle$ and since $R\langle X\rangle$ is coprimely packed, it follows from (v) of our definition that $M R\langle X\rangle=\operatorname{rad}(f R\langle X\rangle)$ for some polynomial $f$ in $R[X]$. (Note that here we have $\operatorname{rad}(I)=j-\operatorname{rad}(I)$ for any nonzero ideal $I$ in $R$ or in $R\langle X\rangle$.) Let $b$ be any element of $M$, and let $a$ be the leading coefficient of $f$, and observe that $a$ is in $M$. Then $b \in M R\langle X\rangle$, and hence there is a positive integer $n$ such that $b^{n}=f(g / h)$ for some $g, h \in R[X]$ with $h$ monic. Thus $b^{n} h=f g$, which implies that $b^{n}=a c$ where $c$ is the leading coefficient of $g$. That is, $b^{n} \in R a$ and therefore $M=\operatorname{rad}(R a)$. Hence by (v) of our definition, $R$ is coprimely packed. 
Note that the above proof works for any integral domain of dimension one.

Theorem 1.2. Let $R$ be a Noetherian normal domain. Then the ring $R\langle X\rangle$ is coprimely packed if and only if $R$ is coprimely packed and of dimension at most one.

Proof. In light of Proposition 1.1, we only need to prove the if part. If $R$ is of dimension zero, then both $R$ and $R\langle X\rangle$ are fields, and hence $R\langle X\rangle$ is coprimely packed. So assume that $R$ is of dimension one. Then both $R$ and $R\langle X\rangle$ are Dedekind domains. Let $N^{*}$ be any maximal ideal of $R\langle X\rangle$. Then $N^{*}$ is the extension of a prime ideal $Q^{*}$ of $R[X]$. Let $Q^{*} \cap R=Q$ in $R$. If $Q$ is maximal in $R$, then $Q^{*}=Q[X]$ in $R[X]$, since otherwise $0 \subset Q[X] \subset Q^{*}$ implies that $Q^{*}$ is of height two in $R[X]$, and hence $Q^{*}=(Q, f)$ for some irreducible monic polynomial $f$ in $R[X]$ (the choice irreducibility of $f$ in $R[X]$ is a consequence of the fact that $R$ is normal), a contradiction to the fact that $Q^{*}$ survives in $R\langle X\rangle$. Since $R$ is coprimely packed by [2. Theorem 1.2], there is an element $a$ in $Q$ such that $R a=Q^{n}$, for some positive integer $n$. But then it is clear that $N^{*^{n}}=(Q R\langle X\rangle)^{n}=R\langle X\rangle a$. We now consider the case when $Q=Q^{*} \cap R=0$ in $R$. By [7] Theorem 36], $Q^{*}$ corresponds to a prime ideal of $K[X]$, where $K$ is the field of fractions of $R$. Therefore $Q^{*} K[X]=g K[X]$ for some polynomial $g$, which we may choose to be in $Q^{*}$. Let $A_{g}$ be the content ideal of $g$ in $R$. Then $\left(A_{g}\right)^{m}=R b$ for some positive integer $m$ and some $b$ in $A_{g}$. Since $R$ is a Dedekind domain by [6], we have $A_{f g}=A_{f} A_{g}$ for any two polynomials $f$ and $g$ in $R[X]$. Hence it follows that $A_{g^{m}}=\left(A_{g}\right)^{m}=R b$. Let $g^{m}=c_{0}+c_{1} X+\cdots+c_{s} X^{s}$. Then $c_{i}=b r_{i}(0 \leq i \leq s)$ and $b=c_{0} d_{0}+c_{1} d_{1}+\cdots+c_{s} d_{s}=b\left(r_{0} d_{0}+r_{1} d_{1}+\cdots+r_{s} d_{s}\right)$, which implies that $r_{0} d_{0}+r_{1} d_{1}+\cdots+r_{s} d_{s}=1$. Thus if $h=r_{0}+r_{1} X+\cdots+r_{s} X^{s}$, then $g^{m}=b h$ and $A_{h}=R$. Since $g^{m}=b h \in Q^{*}$ and $Q^{*} \cap R=0$, we obtain $h \in Q^{*}$. Clearly $h$ is not contained in any other height one prime ideal of $R[X]$ of the form $M[X]$ where $M$ is a maximal ideal of $R$. Also, since $g^{m} K[X]=b h K[X]=h K[X]$, it follows that $h$ is not contained in any other prime $P^{*}$ with $P^{*} \cap R=0$. Thus $Q^{*}$ is the only height one prime ideal of $R[X]$ containing $h$. Hence $N^{*}=Q^{*} R\langle X\rangle$ is the only maximal ideal in $R\langle X\rangle$ containing $h R\langle X\rangle$. But then $N^{*^{n}}=h R\langle X\rangle$ for some positive integer $n$, proving that $R\langle X\rangle$ is coprimely packed.

\section{Coprime PACKEDNESS AND SET TheOREtic COMPlete INTERSECtions}

Recall that an ideal $I$ of a $\operatorname{ring} R$ is a complete intersection if $\operatorname{ht}(I)=\mu(I)$, where $\mathrm{ht}(I)$ is the height of $I$, the infimum of the heights of prime divisors of $I$ and $\mu(I)$ is the minimum number of generators of $I$. The ideal $I$ is said to be a set theoretic complete intersection if $\operatorname{rad}(I)=\operatorname{rad}\left(a_{1}, a_{2}, \ldots, a_{n}\right)$ where $n=\operatorname{ht}(I)$.

Theorem 2.1. For a Noetherian normal domain $R$, the following statements are equivalent.

(a) $R\langle X\rangle$ is coprimely packed.

(b) $R\langle X\rangle$ is a Dedekind domain with torsion ideal class group.

(c) $R$ is a Dedekind domain with torsion ideal class group.

(d) $R$ is of dimension one, and each proper prime ideal of $R[X]$ is a set theoretic complete intersection.

Proof. $(\mathrm{a}) \Leftrightarrow(\mathrm{b}) \Leftrightarrow(\mathrm{c})$. Follows from Theorem[1.2 above and Theorem 1.4 of [2]. 
$(c) \Rightarrow(d)$. From the proof of Theorem 1.2 we have that each height one prime ideal of $R[X]$ is the radical of a principal ideal. If, on the other hand, $M^{*}$ is a height two prime ideal of $R[X]$, then $M^{*}=(M, f)$, for some maximal ideal $M$ in $R$ and some irreducible monic polynomial $f$ in $R[X]$. But in $R, M^{n}=R a$ for some positive integer $n$. Therefore it follows that $M^{*}=\operatorname{rad}(a, f)$ in $R[X]$, and so $M^{*}$ is a set theoretic complete intersection.

(d) $\Rightarrow$ (a). Since each nonzero prime ideal $N^{*}$ of $R\langle X\rangle$ is an extension of a prime ideal $Q^{*}$ of $R[X]$ of height one and since $Q^{*}=\operatorname{rad}(f)$ in $R[X]$, it follows that $N^{*}=Q^{*} R\langle X\rangle=\operatorname{rad}(f)$ in $R\langle X\rangle$, and so $R\langle X\rangle$ is coprimely packed.

Remark 2.2. It is not always the case that if $R$ is a coprimely packed Noetherian integral domain, then each proper prime ideal of $R[X]$ is a set theoretic complete intersection. For let $K$ be a field of characteristic zero and $R$ be the subring of $K[[Y]]$ consisting of those power series whose $Y$ term has coefficient zero. Then it is shown in [5 p. 6469] that $R$ is a local Noetherian integral (not normal) domain of dimension one, and there is a height one maximal ideal in $R[X]$ that is not the radical of a principal ideal.

Remark 2.3. We now note that if $R$ is Artinian, then $R, R[X], R\langle X\rangle, R(X)$ are all coprimely packed, and that if $I^{*}$ is any ideal of $R[X]$, then $\operatorname{rad}\left(I^{*}\right)=\operatorname{rad}(f)$ for some element $f$ in $I^{*}$. For if $N R$ is the nil-radical of $R$, then $N R[X]$ is contained in every prime ideal of $R[X]$. But $R[X] / N R[X] \approx(R / N R)[X]$ is a principal ideal ring. Therefore if $I^{*}$ is any ideal of $R[X]$, the image of $I^{*}$ in $(R / N R)[X]$ is principal. Let $f$ be the element of $I^{*}$ whose image in $(R / N R)[X]$ generates the image of $I^{*}$ in $(R / N R)[X]$. But then it follows that $\operatorname{rad}\left(I^{*}\right)=\operatorname{rad}(f)$.

Theorem 2.4. Over a Noetherian arithmetical ring $R$, the following statements are equivalent.

(a) $R$ is coprimely packed.

(b) $R\langle X\rangle$ is coprimely packed.

(c) Each proper prime ideal of $R[X]$ is a set theoretic complete intersection.

Proof. (a) $\Rightarrow$ (b). We first note that a Noetherian arithmetical ring $R$ is of the form $\prod_{i=1}^{n} R_{i}$ where each factor $R_{i}$ is either a Dedekind domain or an Artin principal ideal ring. Since $R$ is coprimely packed, it follows that each factor $R_{i}$ is coprimely packed. Hence in case $R_{i}$ is a Dedekind domain, by Theorem 2.1, $R_{i}\langle X\rangle$ is coprimely packed. If, on the other hand, $R_{i}$ is an Artin principal ideal ring, then $R_{i}\langle X\rangle$ is a zero-dimensional Noetherian ring and hence is semi local and therefore is coprimely packed. Thus each prime ideal in $R_{i}\langle X\rangle(1 \leq i \leq n)$ is the radical of a principal ideal. But then the same is true for each prime ideal of $R\langle X\rangle=\prod_{i=1}^{n} R_{i}\langle X\rangle$. Therefore $R\langle X\rangle$ is coprimely packed.

(b) $\Rightarrow$ (a). If $R\langle X\rangle=\prod_{i=1}^{n} R\langle X\rangle$ is coprimely packed, then each factor $R_{i}\langle X\rangle$ is coprimely packed. Hence if $R_{i}$ is a Dedekind domain, then by Theorem 2.1, $R_{i}$ is coprimely packed. But if $R_{i}$ is Artinian, then it is coprimely packed. Hence using the above argument we see that $R=\prod_{i=1}^{n} R_{i}$ is coprimely packed.

(a) $\Rightarrow$ (c). Since $R[X]=\prod_{i=1}^{n} R_{i}[X]$, it follows from Theorem [2.1, and Remark 2.3 that each proper prime ideal of each factor $R_{i}[X]$ is a set theoretic complete intersection. But this is so if and only if each proper prime ideal of $R[X]$ is a set theoretic complete intersection.

(c) $\Rightarrow$ (b). Follows from the proof of $(d) \Rightarrow$ (a) of Theorem 2.1 and the first sentence in Remark 2.3. (We argue componentwise.) 
Theorem 2.5. For a Bézout domain $R$ of dimension one the following statements are equivalent.

(a) $R$ is coprimely packed.

(b) $R\langle X\rangle$ is coprimely packed.

(c) Each proper prime ideal of $R[X]$ is a set theoretic complete intersection.

Proof. We first recall that an integral domain $R$ is Bézout if each finitely generated ideal of $R$ is principal, and that over a Bézout domain $R, \operatorname{dim} R[X]=\operatorname{dim} R+1$. Hence by 8 , Theorem 2.1, it follows that $R\langle X\rangle$ is of dimension one.

(a) $\Rightarrow$ (b). The proof is essentially the same as that of Theorem 1.2. Let $N^{*}$ be any maximal ideal of $R\langle X\rangle$. Then $N^{*}$ is the extension of a height one prime ideal $Q^{*}$ of $R[X]$. If $Q^{*} \cap R=Q$ and $Q=\operatorname{rad}(a)$ in $R$, then it follows from the proof of Theorem 1.2 that $N^{*}=\operatorname{rad}(a)$ in $R\langle X\rangle$. If, on the other hand, $Q^{*} \cap R=0$ in $R$, then $Q^{*} K[X]=g K[X]$, where $K$ is the field of fractions of $R$ and $g$ is a polynomial in $Q^{*}$. Since $R$ is Bézout, the content ideal $A_{g}$ of $g$ in $R$ is principal. Hence using the proof of Theorem 1.2, we see that there is a polynomial $h$ in $Q^{*}$ that is a factor of $g$ with $A_{h}=R$ and $Q^{*}=\operatorname{rad}(h)$. But then it follows that $N^{*}=Q^{*} R\langle X\rangle=\operatorname{rad}(h R\langle X\rangle)$. Therefore $R\langle X\rangle$ is coprimely packed.

(b) $\Rightarrow$ (a). The same as that of the proof of Proposition 1.1

(a) $\Rightarrow$ (c). Let $Q^{*}$ be any nonzero prime ideal of $R[X]$. Then either $Q^{*} \cap R=0$ or $Q^{*} \cap R=Q$, a prime ideal of $R$. If $Q^{*} \cap R=0$, then using the proof of (a) $\Rightarrow$ (b) we have $Q^{*}=\operatorname{rad}(h)$, for some polynomial $h$ in $Q^{*}$ with $A_{h}=R$. If, on the other hand, $Q^{*} \cap R=Q$, then either $Q^{*}=Q R[X]$ or $Q^{*}$ is of height two and therefore $Q^{*}=(Q, f)$, for some irreducible monic polynomial $f$ in $R[X]$. Now because $R$ is coprimely packed, $Q=\operatorname{rad}(a)$ for some $a \in Q$. But then it is clear that in $R[X]$, either $Q^{*}=\operatorname{rad}(a)$ when it is of height one, or $Q^{*}=\operatorname{rad}(a, f)$ when it is of height two, showing that $Q^{*}$ is a set theoretic complete intersection.

(c) $\Rightarrow($ b). The same as that of the proof of $(d) \Rightarrow$ (a) of Theorem 2.1

\section{REFERENCES}

1. J. W. Brewer and W. J. Heinzer, $R$ Noetherian implies $R\langle X\rangle$ is a Hilbert ring, J. Algebra, 67 (1980), 204-209. MR 82d:13010

2. V. Erdoğdu, Coprimely packed rings, J. Number Theory, 28 (1988), 1-5. MR 89f:13025

3. V. Erdoğdu, The prime avoidance of ideals in Noetherian Hilbert rings, Communications in Algebra, 22 (1994), 4989-4990.

4. V. Erdoğdu, Three notes on coprime packedness, J. Pure Appl. Algebra, 148 (2000), 165-170. MR 2001b:13004

5. V. Erdoğdu and S. McAdam, Coprimely packed Noetherian polynomial rings, Communications in Algebra, 22 (1994), 6459-6470. MR 95k:13025

6. S. Glaz and W. Vasconcelos, The content of Gaussian polynomials, J. Algebra, 202 (1998), 1-9. MR 99c:13003

7. I. Kaplansky, Commutative Rings, University of Chicago Press, 1974. MR 49:10674

8. L. Le Riche, The ring $R\langle X\rangle$, J. Algebra, 67 (1980), 327-341. MR 82d:13011

9. D. E. Rush, Generating ideals up to radical in Noetherian polynomial rings, Communications in Algebra, 25 (1997), 2169-2191. MR 98i:13041

Department of Mathematics, Istanbul Technical University, Maslak, 80626 Istanbul, TURKEY

E-mail address: erdogdu@itu.edu.tr 